\title{
Ten-Year Results of a Randomized Trial Comparing Tacrolimus Versus Cyclosporine A in Combination With Mycophenolate Mofetil After Heart Transplantation
}

\author{
Sonja Guethoff, ${ }^{1,3}$ Bruno M. Meiser, ${ }^{2}$ Jan Groetzner, ${ }^{1}$ Sandra Eifert, ${ }^{1}$ Carola Grinninger, ${ }^{2}$ Peter Ueberfuhr, ${ }^{1}$ \\ Bruno Reichart, ${ }^{1}$ Christian Hagl, ${ }^{1}$ and Ingo Kaczmarek ${ }^{1,2}$
}

\begin{abstract}
Background. Long-term results of prospective randomized trials comparing triple immunosuppressive strategies combining tacrolimus (TAC) or cyclosporine A (CsA) with mycophenolate mofetil (MMF) and steroids after heart transplantation (HTX) are rarely published. Therefore, we collected long-term follow-up data of an intervention cohort 10 years after randomization.

Methods. Ten-year follow-up data of 60 patients included in a prospective, randomized trial between 1998 and 2000 were analyzed as intention-to-treat (TAC-MMF n=30; CsA-MMF $n=30$ ). Baseline characteristics were well balanced. Cardiac allograft vasculopathy (CAV) was graduated in accordance with the new ISHLT classification.

Results. Survival at 1,5 , and 10 years was $96.7 \%, 80.0 \%$, and $66.7 \%$ for TAC-MMF and $90.0 \%, 83.3 \%$, and $80.0 \%$ for CsA-MMF $(P=n s)$. Freedom from acute rejection (AR) was significantly higher in TAC-MMF versus CsA-MMF (65.5\% vs. $21.7 \%$, log-rank 8.3, $P=0.004$ ). Freedom from ISHLT $\geq \mathrm{CAV}_{1}$ after 5 and 10 years was in TAC-MMF $64.0 \%$ and 45.8\%, and in CsA-MMF 36.0\% (log-rank 3.0, $P=0.085$ ) and $8.0 \%$ (log-rank 9.0, $P=0.003$ ). No difference in longterm results for freedom from coronary angioplasty or stenting, renal dysfunction, diabetes mellitus, CMV infection, or malignancy was detected.

Conclusion. Cross-over effects because of treatment switch may result in impairment of significance between the groups. The long-term analysis resulted in a significant difference in manifestation of CAV between the groups after 10 years. Less rejection in the TAC-group might have contributed to the lower incidence of CAV. Superior freedom from AR and CAV in the TAC-MMF group did not result in better long-term survival.
\end{abstract}

Keywords: Heart transplantation, Tacrolimus, Cyclosporine A, Cardiac allograft vasculopathy, Long-term.

(Transplantation 2013;95: 629-634)

$\mathrm{S}^{\mathrm{i}}$ ince TAC was introduced into the clinic in 1989 (1-5), it progressively replaced CsA as calcineurin inhibitor $(\mathrm{CNI})$ after HTX (6). One of the first prospective, randomized

Only for the first 2 years of follow-up the randomized study was investigator driven and supported by grants from Fujisawa GmbH (Munich, Germany), Novartis AG (Basel, Switzerland), and Roche Pharmaceuticals (Basel, Switzerland). There was no conflict of interest or funding sources about the long-term results based on routine examinations.

${ }^{1}$ Department of Cardiac Surgery, University of Munich (LMU), Munich, Germany.

2 Transplantation Center Munich, University of Munich (LMU), Munich, Germany.

3 Address correspondence to: Sonja Guethoff, Department of Cardiac Surgery, University of Munich (LMU), Marchioninistr. 15, 81377 München, Germany.

E-mail: Sonja.Guethoff@med.uni-muenchen.de

S.G., S.E., C.G., C.H., and I.K. participated in the writing of the paper; B.M.M., J.G., P. U., B.R., and I.K. participated in the performance of the initial trial; and S.G. and I.K. participated in data analysis.

Received 29 August 2012. Revision requested 19 September 2012.

Accepted 5 October 2012.

Copyright (C) 2013 by Lippincott Williams \& Wilkins

ISSN: 0041-1337/13/9504-629

DOI: $10.1097 /$ TP.0b013e318277e378

Transplantation • Volume 95, Number 4, February 27, 2013 studies comparing CsA and TAC after HTX demonstrated both CNI with the same efficacy and safety but with a trend to less acute rejections for treatment with TAC (7). MMF, clinically approved in 1995, was associated with a superior prevention of acute rejection compared with Azathioprine (AZA) in combination with CsA and corticosteroids (CS) in renal transplant recipients $(8)$. The improvement of 1 -year mortality and less acute rejection during the first year after HTX was verified for MMF as a substitute for AZA (9). In 1998, we designed a prospective randomized study to determine whether TAC or CsA is the better partner for MMF after HTX (10). The results 2 years after randomization presented both $\mathrm{CNI}$ as adequate and effective partners with MMF to prevent acute rejection in heart transplant recipients. In patients treated with TAC-MMF, significantly fewer rejections were observed. Patients treated with CsA-MMF needed significantly higher doses of MMF to achieve the MPA target levels. With respect to CAV, no significant difference between the treatment groups was discovered; however, a more pronounced intimal proliferation was detected in the CsA-MMF group. Despite no differences in 2-year survival, we concluded

www.transplantjournal.com 
that the short-term results of this randomized trial indicate the superiority of TAC over CsA as better partner for MMF after HTX (10).

Other prospective, randomized studies comparing TAC and CsA (10-20) also presented short- or mid-term results based on follow-up periods from 6 months to 3 years and in one case of 5 years (17).

The ISHLT registry detected a median survival of 11 years for the entire cohort of adult and pediatric heart recipients since initiation of the Registry in 1982 (6).

In view of the numerousness of heart recipients who fortunately live more than 5 years after transplantation, the question occurs whether the beneficial early results for TAC observed in our prospective, randomized study could be confirmed with longer follow-up and whether there will be an impact on long-term outcome.

\section{RESULTS}

\section{Baseline Characteristics and Patient Accounting}

Patient demographic and baseline characteristics shown in Table 1 were equally distributed across both treatment groups. Because of death, the mean follow-up period was $9.1 \pm 3.3$ years in TAC-MMF ITT and $8.8 \pm 4.0$ years in CsAMMF ITT $(P=0.712)$. There was no dropout of the randomized 60 patients but missing data in subcategories. Switch to another immunosuppressive drug did not result in exclusion from the ITT analysis. Because one patient of the TAC-group moved to another transplantation center, only his survival data were analyzed. Baseline coronary angiography detected preexisting coronary artery disease in one patient of each group.

\section{Survival}

Causes of death in the TAC-group were cancer $(n=4$, $3.4,4.0,4.3$, and 5.7 years), acute rejection $(n=2,5.6$, and
7.2 years; the second case because of noncompliance in taking medications), systemic aspergillosis $(\mathrm{n}=1,45$ days), sepsis ( $n=1,2.6$ years), ileus ( $n=1,2.0$ years), and multiorgan failure ( $n=1,8.2$ years). Regarding the CsA group, there was death because of cardiovascular complications $(\mathrm{n}=3,190$ days, 2.9 and 3.7 years), systemic aspergillosis $(\mathrm{n}=2,10$ and 12 days) and cancer $(\mathrm{n}=1,6.2$ years $)$.

There was no statistical difference in long-term survival between the treatment groups (Fig. 1). Survival after 1 year was 93.3\% (log-rank 1.1, $P=0.301)$ : in TAC-MMF ITT 96.7\% $(n=29)$, and in CsA-MMF ITT 90.0\% $(n=27)$; after 5 years, $81.7 \%$ (log-rank $0.1, P=0.800)$ : in TAC-MMF ITT $80.0 \%(n=24)$, and in CsA-MMF ITT 83.3\% $(n=25)$; and after 10 years, $73.3 \%$ (log-rank 1.0, $P=0.308$ ): in TACMMF ITT 66.7\% $(n=20)$, and in CsA-MMF ITT $80.0 \%$ $(n=24)$. Patients who had never been switched to another immunosuppressive drug during treatment presented with inferior survival without statistical significance $(69.8 \%, P=0.369)$.

\section{Acute Rejection}

TAC-MMF-treated patients had significantly lower rates of rejection episodes than the CsA-MMF-treated patients as shown in Figure 2. Freedom from acute rejection after 1 year was $47.5 \%$ in the study cohort with considerable benefit of TAC-MMF (log-rank 6.1, $P=0.013$ ): in TACMMF ITT 65.5\% $(n=19)$, and in CsA-MMF ITT 30.0\% $(\mathrm{n}=9)$; after 5 years, $44.1 \%$ (log-rank $8.3, P=0.004)$ : in TACMMF ITT 65.5\% $(n=19)$, and in CsA-MMF ITT 23.3\% $(n=7)$; after 10 years $42.4 \%$ (log-rank 8.2, $P=0.004)$ : in TAC-MMF ITT $65.5 \% \quad(n=19)$, and in CsA-MMF ITT $21.7 \%(n=6)$. Mean freedom from acute rejection was in TAC-MMF ITT $6.6 \mathrm{y} \pm 0.8$ years, and in CsA-MMF ITT $2.9 \pm 0.8$ years (95\% CI $4.9-8.3$ and $1.4-4.4$ years).

Variance analysis on AR onset detected for MPA levels during the first year a trend $(P=0.051)$, and for ischemic time

TABLE 1. Patient demographic and baseline characteristics

Characteristics

No. of patients

Mean follow-up time (yr)

Mean donor age (yr)

Mean recipient age (yr)

Donor sex $(\mathrm{M} / \mathrm{F})$

Recipient sex (M/F)

Sex mismatch (recipient M/F)

Primary diagnosis

Dilated CMP

Ischemic CMP

CMV IgG Donor +

CMV IgG Recipient -

CMV Donor +/Recipient -

CMV prophylaxis postoperative

Mean ischemic time (h)

Operation duration (h)

ICU (d)

Ventilation postoperative (d)
TAC-MMF ITT

CsA-MMF ITT

30

$9.1 \pm 3.3$

$38 \pm 14$

$55 \pm 1$

$16 / 14$

$26 / 4$

$10 / 0$

\section{5}

15

13

13

6

9

$3.4 \pm 1.1$

$4.7 \pm 0.9$

$9.1 \pm 11.1$

$2.5 \pm 2.9$

\begin{tabular}{cl} 
CsA-MMF ITT & \multicolumn{1}{c}{$\boldsymbol{P}$} \\
\hline 30 & 1.0 \\
$8.8 \pm 4.0$ & 0.712 \\
$42 \pm 13$ & 0.297 \\
$55 \pm 12$ & 0.824 \\
$18 / 12$ & 0.795 \\
$24 / 6$ & 0.731 \\
$7 / 1$ & 0.347
\end{tabular}

15

1.0

1.0

0.396

0.5

1.0

0.392

0.281

0.480

0.248

0.627

mean \pm SD; CMP, cardiomyopathy; +, positive; -, negative. 


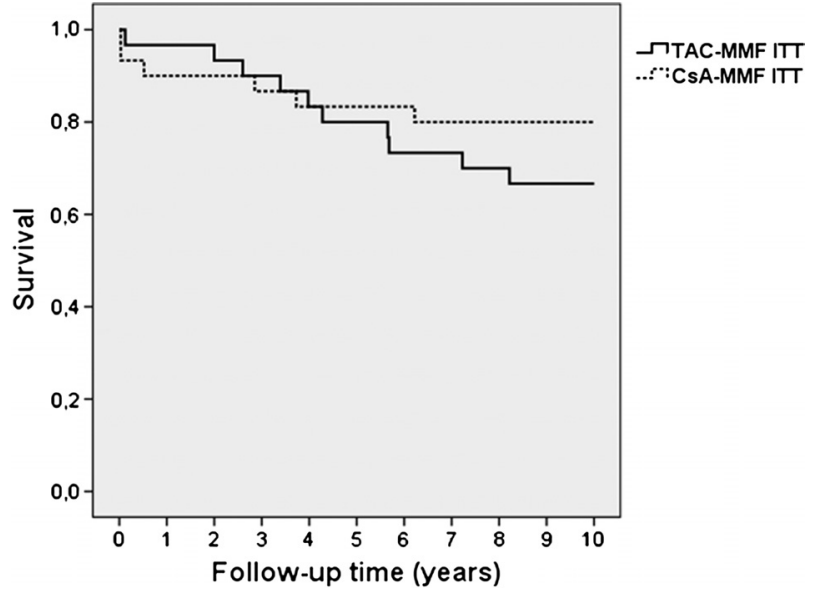

FIGURE 1. Survival: Kaplan-Meier analysis for 10 years after HTX (difference between the ITT groups: log-rank 1.0, $P=0.308)$.

a significant impact $(P=0.027)$. Adjusted for early MPA levels and ischemic time, there was less AR in TAC-MMF ITT group (OR 5.1, 95\% CI 1.4-18.3, $P=0.013$ ). Comparing patients with and without AR within the same ITT, there were no differences in MPA and TAC, respectively, CsA levels. Isolated analysis of patients treated continuously with the randomized immunosuppressive drug $(n=43)$ showed comparable results with a freedom from AR of 46.5\% 10 years after HTX (log-rank 9.8, $P=0.002)$ : in TAC-MMF 68.0\% $(n=17)$, and in CsA-MMF 16.7\% $(n=3)$.

\section{Freedom From Treatment Failure}

Treatment failure resulted in switch to another primary immunosuppressive drug. Four patients (13.8\%) of the TAC group were switched to another immunosuppressive drug, three patients to sirolimus (SIR) because of renal dysfunction $(3.9,4.4$, and 9.4 years), and one patient to CsA because of neuropathy (10 days). In the CsA group, 12 patients

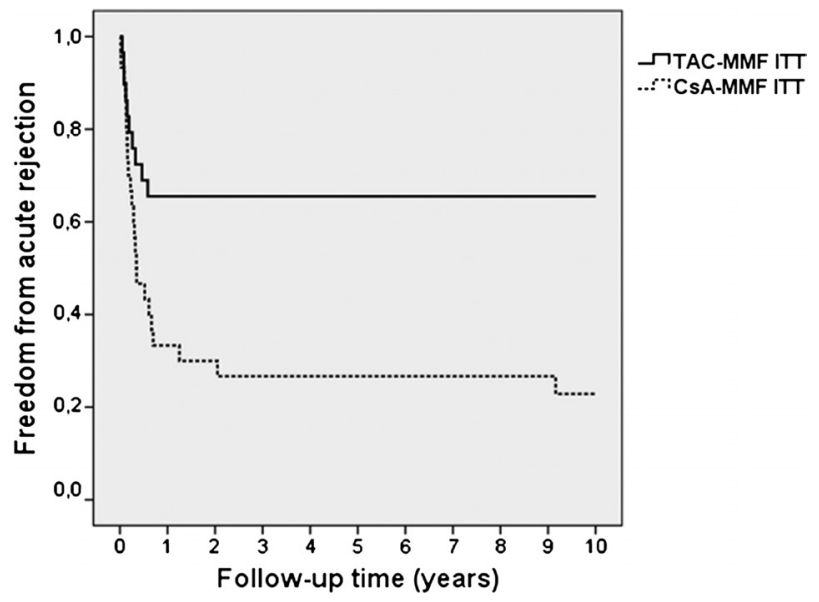

FIGURE 2. Freedom from acute rejection: Kaplan-Meier analysis for 10 years after HTX (difference between the ITT groups: log-rank 8.2, $P=0.004)$.
(40.0\%) were changed, two patients to SIR because of renal dysfunction (1.9 and 3.2 years), five patients to TAC because of $\operatorname{AR}(8,13$, and 67 days, and 1.1 and 1.3 years), one patient to SIR because of AR but renal dysfunction (2.4 years), and one patient in each group to SIR because of neuropathy (4.9 years), to TAC because of gingival hyperplasia ( 1.5 years), to everolimus (EVE) because of CAV (7.0 years) and to TAC because of osteoporosis (2.0 years).

As shown in Figure 3, 1-year freedom from treatment failure was $93.2 \%$ in the study cohort without difference between ITT (log-rank 1.1, $P=0.308$ ): in TAC-MMF ITT 96.6\% $(n=28)$, and in CsA-MMF ITT 93.3\% $(n=27)$; and after 5 years $79.7 \%$ (log-rank 3.7, $P=0.054)$ : in TAC-MMF ITT $89.7 \%(n=26)$, and in CsA-MMF ITT $70.0 \%(n=21)$; and after 10 years, freedom from treatment failure was $72.9 \%$ with significantly less switch in TAC-MMF ITT (logrank 4.8, $P=0.029)$ : in TAC-MMF ITT $86.2 \%(n=25)$, and in CsA-MMF ITT 60.0\% $(\mathrm{n}=18)$.

\section{Cardiac Allograft Vasculopathy}

As shown in Figure 4, freedom from CAV ISHLT $\geq \mathrm{CAV}_{1}$ onset was $96.4 \%$ in the TAC group vs. $88.5 \%$ in the CsA group (log-rank 1.2, $P=0.281$ ) after 1 year, $64.0 \%$ in the TAC group vs. $36.0 \%$ in the CsA group (log-rank 3.0, $P=0.085$ ) after 5 years; and $45.8 \%$ in the TAC group vs. $8.0 \%$ in the CsA group (log-rank 9.0, $P=0.003$ ) after 10 years. The mean time until the first diagnosis of CAV confirmed by coronary angiography was $7.8 \pm 0.8$ years in the TAC group and $4.6 \pm 0.7$ years in the CsA group (95\% CI 6.2-9.4 years and 3.3-5.9 years). Variance analysis on CAV onset could not prove any impact of the common risk parameters such as $\operatorname{AR}(P=0.566)$, donor age $(P=0.124)$, diabetes mellitus $(P=0.805), \mathrm{LDL}$ and cholesterol levels $(P=0.979$ and $P=0.355)$, systemic blood pressure $(P=0.398)$, and positive CMV titer $(P=0.792)$. In contrast, CsA-MMF was a significant risk factor in the multivariate analysis (OR 3.6; 95\% CI 1.1-11.4; $P=0.031$ ).

One patient of the CsA group underwent coronary artery bypass grafting (7.89 years) because of severe CAV. The patient has a simvastatine intolerance.

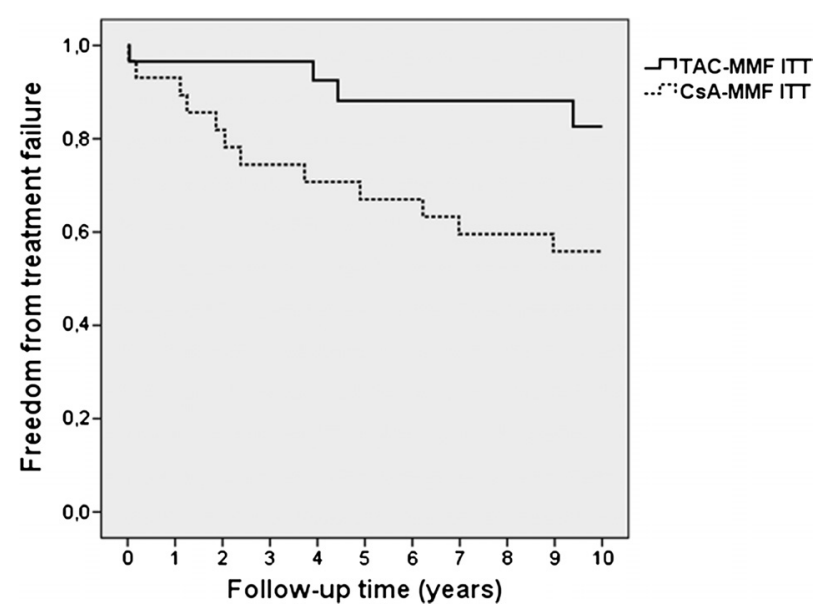

FIGURE 3. Freedom from treatment failure: Kaplan-Meier analysis for 10 years after HTX (difference between the ITT groups: log-rank 4.8, $P=0.029$ ). 


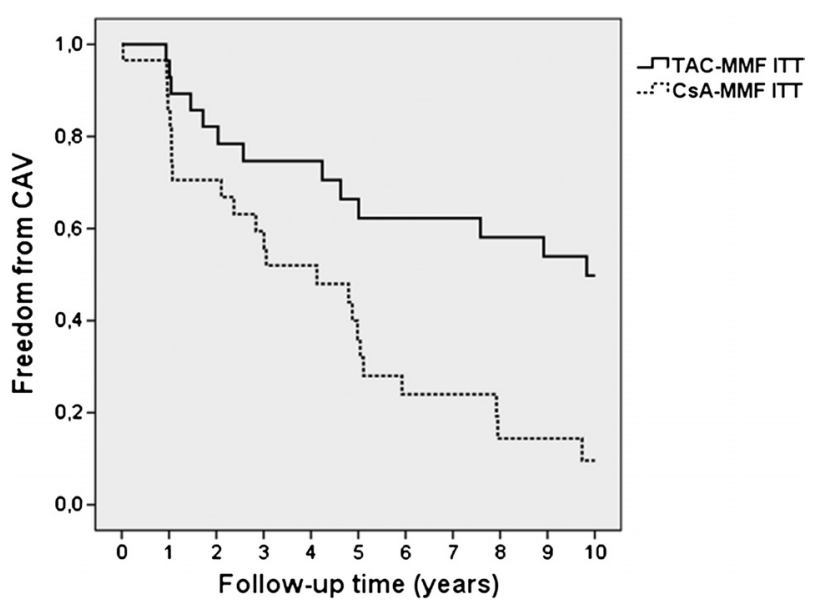

FIGURE 4. Freedom from CAV: Kaplan-Meier analysis for 10 years after HTX (difference between the ITT groups: logrank 9.0, $P=0.003$ ).

Freedom from coronary stenting after follow-up was 87.5\% (log-rank 0.2, $P=0.693$ ).

Regarding the continuous treatment groups without switch to another immunosuppressive drug, there were 11 of TAC-treated patients and only three of CsA-treated patients without CAV after 10 years of follow-up.

\section{Treatment Dosages and Resulted Trough Level}

Within the first 5 years and within the 10 years of follow-up, administered TAC mean dosage of $4.1 \pm 2.0 \mathrm{mg} /$ day and $3.8 \pm 2.2 \mathrm{mg} /$ day resulted in mean trough levels of $10.7 \pm 0.9 \mathrm{ng} / \mathrm{mL}$ and $9.9 \pm 1.0 \mathrm{ng} / \mathrm{mL}$, and a CsA mean dosage of $200 \pm 41 \mathrm{mg} /$ day and $184 \pm 45 \mathrm{mg} /$ day resulted in mean trough levels of $157 \pm 28 \mathrm{ng} / \mathrm{mL}$ and $132 \pm 39 \mathrm{ng} / \mathrm{mL}$. Only within the first 3 months of study duration, the MPA levels (TAC-MMF ITT $3.1 \pm 1.0$ vs CsA-MMF ITT $2.2 \pm 0.6 \mu \mathrm{g} / \mathrm{mL} ; P=0.003)$ were significantly lower in the CsA-MMF-treated patients, despite higher MMF doses.

\section{Cytomegalovirus}

There was no difference between the ITT groups about preoperative CMV status and postoperative CMV prophylaxis (Table 1). Freedom from positive CMV titer was $58.6 \%$ in the TAC group and $73.3 \%$ in the CsA group (log-rank $1.8, P=0.186)$. In two cases of the TAC-MMF ITT and three cases of CsA-MMF ITT, there were severe CMV infections, which required hospital admission. There were no causes of death related to CMV infections.

\section{Adverse Effects}

Only at 5-year follow-up, there was a significantly higher creatinine detected in the CsA group $(1.60 \pm 1.21 \mathrm{mg} / \mathrm{dL}$ in TAC-MMF ITT vs. $1.65 \pm 0.39 \mathrm{mg} / \mathrm{dL}$ in CsA-MMF ITT, $P=0.014$ ), which cannot be reconfirmed at 10 -year follow-up $(1.45 \pm 0.62 \mathrm{mg} / \mathrm{dL}$ in TAC-MMF ITT vs. $1.75 \pm 0.65 \mathrm{mg} / \mathrm{dL}$ in CsA-MMF ITT, $P=0.120)$. After 5 and 10 years, creatinine levels of $2.0 \mathrm{mg} / \mathrm{dL}$ or greater were found in $13.0 \%$ and $22.2 \%$ in the TAC group and in $28.0 \%$ $(P=0.180)$ and $41.7 \%(P=0.161)$ in the CsA group. Permanent hemodialysis was inevitable in one patient of the CsA group. Within the group of patients with drug application as randomized over the whole time of follow-up, there seemed to be a benefit for TAC-MMF over CsA-MMF in long-term creatinine measurement (5-year follow-up: $1.39 \pm 0.61 \mathrm{mg} / \mathrm{dL}$ vs. $1.74 \pm 0.36 \mathrm{mg} / \mathrm{dL}, P=0.005$; and 10 -year follow-up: $1.34 \pm 0.61 \mathrm{mg} / \mathrm{dL}$ vs. $1.91 \pm 0.63 \mathrm{mg} / \mathrm{dL}, P=0.012)$, despite comparable baseline creatinine levels $(1.46 \pm 0.91 \mathrm{mg} / \mathrm{dL}$ vs. $1.15 \pm 0.23 \mathrm{mg} / \mathrm{dL}, P=0.298)$.

Antidiabetic treatment (oral and/or insulin) was necessary in $13.6 \%$ in the TAC group and in $16.7 \%$ in the CsA group $(P=0.551)$ at 5 -year follow-up, and in $22.2 \%$ and in $18.2 \%(P=0.528)$ at 10 -year follow-up.

During follow-up, 16 patients (27.1 \%) developed malignancy without statistical difference between the ITT $(P=0.252) ; 10$ patients $(34.5 \%)$ in TAC-MMF ITT and 6 patients $(20.0 \%)$ in CsA-MMF ITT. Neoplasm of the skin was found in five patients in the TAC group and three patients in the CsA group, lymphoma in one case in each group, and solid tumors in four patients in the TAC group and two patients in the CsA group.

Immunosuppressant-associated side effects were as follows: gastrointestinal disorders in the three cases in the TAC group and one case in the CsA group, neuropathy in two cases in the TAC group and three cases in the CsA group, and osteoporosis in two cases in the TAC group and four cases in the CsA group. In TAC-MMF ITT, one patient experienced restless leg syndrome, and four patients in the CsA group developed gingival hyperplasia.

\section{DISCUSSION}

The study (10) was primarily designed for the first 2 years after HTX. This long-term results are predicated on routine examinations during follow-up period. Regarding long-term survival, there was no significant difference between the treatment groups. Because of the small study cohort, the study may be underpowered to detect differences in survival. Based on ITT analysis, it has to be considered that the additive shorter patient-years for randomized treatment with CsA versus TAC (190 versus 211 patientyears) improved long-term outcome including long-term survival by early switch to an individual beneficial primary immunosuppressive drug.

The definition of AR as ISHLT (21) grade II (2R) and higher or grade IB (1R) combined with clinical symptoms, which necessitated steroid therapy is based on clinical experiences in our transplantation center and was suitable to other randomized studies $(14,16)$. The benefit of TAC over CsA preventing AR after HTX was presented before $(13,14$, $16,18,20)$. The ISHLT stated in the recommendations on the principles of immunosuppressive regimens in heart transplant recipients that the result of clinical trials suggest that TAC-based regimens may be associated with lower rejection rates but not with superior survival compared with CsA-based regimens (22). Our results emphasize this recommendation even with long-term results 10 years after HTX. The lower MPA level during the first 3 months after HTX in CsA-treated patients and the relatively high TAC levels maintained throughout the first year $(11.8 \pm 1.9 \mathrm{ng} / \mathrm{mL})$ may have contributed the significant difference in freedom from AR between the ITT groups. Antibody-mediated rejection was not routinely tested at our center. 
The development of CAV is deemed to be one of the leading causes of posttransplant mortality particularly with increasing follow-up time: between 1 and 3 years after HTX, approximately $10 \%$ of deaths were shown to be associated with CAV (6), and 5 years after HTX, 32\% of deaths were caused by CAV in addition with late-onset graft failure, which could be related to undetected $\operatorname{CAV}(23,24)$. Prospective, randomized studies comparing TAC- and CsAbased immunosuppressive regimens after HTX reported a trend toward superior of preventing $\operatorname{CAV}$ for $\operatorname{TAC}(25,26)$. However, no significant difference between both CNI regimens in angiographically detected CAV could be documented 5 years after $\operatorname{HTX}(17,27)$. Reichenspurner (28) characterized CAV as histologic manifestation of chronic rejection after HTX, and he recognized the lack of long-term data for incidence of CAV in TAC- and CsA-treated heart recipients. In fact, and contrary to the current opinion because of absent evidence (27), our randomized study could unmask a significantly better freedom from CAV in the TAC-MMF ITT group but not until 10 years after HTX.

The duration and array of rejection episodes were described as risk for CAV development $(29,30)$. Both were not determined in this study. Stoica et al. (31) demonstrated that acute moderate and severe cellular rejection had a cumulative impact on CAV onset, whereas mild, untreated rejection was not associated with CAV. This subdivision was not considered in our study.

After 5 years of follow-up, there was a better creatinine level in TAC-MMF-treated patients, and almost twice the number of patients in CsA-MMF group had creatinine levels of $2.0 \mathrm{mg} / \mathrm{dL}$ or greater. Analysis of continuous randomized treatment indicated that TAC-MMF treatment resulted in lower creatinine levels, but a selection bias must be considered. Although the study cohort may be undersized to show differences between the treatment groups, we disclose a trend for TAC-MMF as beneficial therapy in renal function like presented by Kobashigawa et al. (18).

\section{CONCLUSION}

In 2000, 23\% of heart recipients achieved TAC; in 2009 , its use increased to $73 \%$, whereas the use of CsA has decreased below 20\% (6). There is no general preference about CsA or TAC as CNI-based therapy after HTX in the current ISHLT recommendations (22). Furthermore, no evidence of different CAV rates between CsA- and TACbased therapies is documented by the ISHLT (27). We conclude that the lack of evidence is based on insufficient follow-up duration of randomized studies published so far. Despite the limitation because of the small study cohort, this study represents the first published prospective, randomized study comparing TAC versus CsA in combination with MMF over 10 years of follow-up including the new ISHLT nomenclature for CAV (32). Our long-term analysis resulted in a significant difference in manifestation of CAV between TAC-MMF- and CsA-MMF-treated patients. However, cross-over effects because of treatment switch may result in impairment of significance between the groups. However, superior freedom from AR and CAV in the TAC group did not result in better long-term survival.

\section{MATERIALS AND METHODS}

\section{Patients}

Complete 10-year follow-up data of 60 adult orthotopic heart transplant recipients of a prospective, open-label, single-center, randomized trial were analyzed. Between 1998 and 2000, all patients were 1:1 randomized to treatment with TAC $(n=30)$ or CsA $(n=30)$ both in combination with MMF as described previously (10). Exclusion criteria were age younger than 18 years, pregnancy or nursing, unwillingness or inability to use adequate contraceptions during the study, cardiac retransplantation, previous or multiorgan transplantation, human immunodeficiency virus-positive donor or recipient, serum creatinine greater than $2.5 \mathrm{mg} / \mathrm{dL}$ or elevated transaminases greater than 1.5 times above reference value, or participation in any other investigational drug study within 28 days of study entry. The study protocol was approved by the local ethics committee and was conformed to the Declaration of Helsinki. All patients gave written informed consent before inclusion.

University of Wisconsin solution was used as preservation medium. Patients underwent routine follow-up examinations including drug level monitoring, transvenous endomyocardial biopsy, echocardiography, and coronary angiography according to center practice.

\section{Study Medication and Drug Monitoring}

Study medication (initial intravenously, subsequent oral) was administered as described previously (10). Both CNIs and MMF were adjusted to target levels (TAC: month $0-6=13-15 \mathrm{mg} / \mathrm{mL}$, month $7-12=10-12 \mathrm{mg} / \mathrm{mL}$, second year $=8-10 \mathrm{mg} / \mathrm{mL}$, third to fourth year $=6-8 \mathrm{mg} / \mathrm{mL}$, fifth to tenth year= $4-7 \mathrm{mg} / \mathrm{mL}$; CsA: month 0-6=200-300 mg/mL, month 7-24=150-200 mg/mL, third to tenth year $=100-150 \mathrm{mg} / \mathrm{mL}$; MPA: month $0-6=2.5-4.0 \mu \mathrm{g} / \mathrm{mL}$, month 7 to tenth year: $1.5-2.5 \mu \mathrm{g} / \mathrm{mL}$ ).

All patients received an intraoperative bolus of $500 \mathrm{mg}$ methylprednisolone intravenously followed by $3 \times 125 \mathrm{mg}$ within the initial 24 hours after HTX. Prednisolone was weaned standardized without difference between the treatment groups; the therapy was given for 6 months and withdrawn in patients without repeated rejection episodes. None of the patients received induction therapy. Because statins are beneficial for survival after HTX (33), all patients received simvastatine routinely at a minimum dose of $5 \mathrm{mg} /$ day or in case of persistent hypercholesterolemia (total cholesterol, $>200 \mathrm{mg} / \mathrm{dL}$ or low-density lipoprotein cholesterol $>140 \mathrm{mg} / \mathrm{dL}$ ) at a maximum dosage of $20 \mathrm{mg} /$ day.

\section{Acute Rejections}

Transvenous endomyocardial biopsies were performed routinely after 1 , 2, 3, 4, 6, 8, and 10 weeks; after 3, 4, 5, 6, 9, 12, and 24 months; and when clinically indicated. Rejection was defined in adherence with the ISHLT (21) as histologic grade II (2R) and higher or grade IB (1R) combined with clinical symptoms, which necessitated steroid therapy or any treated rejection. According to the protocol in patients experiencing three rejection episodes, a switch of the primary immunosuppressant was mandatory.

\section{Cardiac Allograft Vasculopathy}

Diagnosis of CAV was based on coronary angiography. There was a baseline coronary angiography 1 month after transplantation for detecting preexisting coronary artery disease. Afterward, the examinations were carried out annually during the first years and increasingly infrequent when clinically not indicated. CAV was graduated in accordance with the new nomenclature for CAV published by the ISHLT in 2010 (32).

\section{Statistical Analysis}

Data are presented as ITT analysis unless otherwise indicated. Survival, freedom from acute rejection, treatment switch, and CAV were calculated using Kaplan-Meier analysis, and differences were assessed using log-rank test. As nonparametric test, the Wilcoxon rank-sum test was used. The chisquare test was performed to evaluate differences in adverse events. Influence of risk factors on AR and CAV onset were analyzed using logistic regression analysis. Numerical data are listed as mean \pm standard deviation (SD). A $P<0.05$ was considered significant. 


\section{REFERENCES}

1. Starzl TE, Todo S, Fung J, et al. FK 506 for liver, kidney, and pancreas transplantation. Lancet 1989; 2: 1000.

2. Todo S, Fung JJ, Demetris AJ, et al. Early trials with FK 506 as primary treatment in liver transplantation. Transplant Proc 1990; 22: 13.

3. Starzl TE, Fung J, Jordan M, et al. Kidney transplantation under FK 506. JAMA 1990; 264: 63

4. Armitage JM, Kormos RL, Fung J, et al. Preliminary experience with FK506 in thoracic transplantation. Transplantation 1991; 52: 164.

5. Armitage JM, Kormos RL, Fung J, et al. The clinical trial of FK 506 as primary and rescue immunosuppression in adult cardiac transplantation. Transplant Proc 1991; 23: 3054.

6. Stehlik J, Edwards LB, Kucheryavaya AY, et al. The Registry of the International Society for Heart and Lung Transplantation: Twentyeighth Adult Heart Transplant Report-2011. J Heart Lung Transplant 2011; 30: 1078 .

7. Meiser BM, Uberfuhr P, Fuchs A, et al. Single-center randomized trial comparing tacrolimus (FK506) and cyclosporine in the prevention of acute myocardial rejection. J Heart Lung Transplant 1998; 17: 782.

8. Fulton B, Markham A. Mycophenolate mofetil. A review of its pharmacodynamic and pharmacokinetic properties and clinical efficacy in renal transplantation. Drugs 1996; 51: 278.

9. Kobashigawa J, Miller L, Renlund D, et al. A randomized activecontrolled trial of mycophenolate mofetil in heart transplant recipients. Mycophenolate Mofetil Investigators. Transplantation 1998; 66: 507.

10. Meiser BM, Groetzner J, Kaczmarek I, et al. Tacrolimus or cyclosporine: which is the better partner for mycophenolate mofetil in heart transplant recipients? Transplantation 2004; 78: 591.

11. Reichart B, Meiser B, Vigano M, et al. European Multicenter Tacrolimus (FK506) Heart Pilot Study: one-year results-European Tacrolimus Multicenter Heart Study Group. J Heart Lung Transplant 1998; 17: 775.

12. Taylor DO, Barr ML, Radovancevic B, et al. A randomized, multicenter comparison of tacrolimus and cyclosporine immunosuppressive regimens in cardiac transplantation: decreased hyperlipidemia and hypertension with tacrolimus. J Heart Lung Transplant 1999; 18: 336.

13. Mehra MR, Uber PA, Scott RL, et al. Ethnic disparity in clinical outcome after heart transplantation is abrogated using tacrolimus and mycophenolate mofetil-based immunosuppression. Transplantation 2002; 74: 1568.

14. Wang CH, Ko WJ, Chou NK, et al. Efficacy and safety of tacrolimus versus cyclosporine microemulsion in primary cardiac transplant recipients: 6-month results in Taiwan. Transplant Proc 2004; 36: 2384.

15. Pollock-Barziv SM, Dipchand AI, McCrindle BW, et al. Randomized clinical trial of tacrolimus- vs cyclosporine-based immunosuppression in pediatric heart transplantation: preliminary results at 15-month follow-up. J Heart Lung Transplant 2005; 24: 190.

16. Grimm M, Rinaldi M, Yonan NA, et al. Superior prevention of acute rejection by tacrolimus vs. cyclosporine in heart transplant recipients-a large European trial. Am J Transplant 2006; 6: 1387.

17. Kobashigawa JA, Patel J, Furukawa H, et al. Five-year results of a randomized, single-center study of tacrolimus vs microemulsion cyclosporine in heart transplant patients. J Heart Lung Transplant 2006; 25: 434.
18. Kobashigawa JA, Miller LW, Russell SD, et al. Tacrolimus with mycophenolate mofetil (MMF) or sirolimus vs. cyclosporine with MMF in cardiac transplant patients: 1-year report. Am J Transplant 2006; 6: 1377.

19. Wang SS, Chou NK, Chi NH, et al. Heart transplantation under cyclosporine or tacrolimus combined with mycophenolate mofetil or everolimus. Transplant Proc 2008; 40: 2607.

20. Sanchez-Lazaro IJ, Almenar L, Martinez-Dolz L, et al. A prospective randomized study comparing cyclosporine versus tacrolimus combined with daclizumab, mycophenolate mofetil, and steroids in heart transplantation. Clin Transplant 2011; 25: 606.

21. Billingham ME, Cary NR, Hammond ME, et al. A working formulation for the standardization of nomenclature in the diagnosis of heart and lung rejection: Heart Rejection Study Group. The International Society for Heart Transplantation. J Heart Transplant 1990; 9: 587.

22. The International society of heart and lung transplantation guidelines for the care of heart transplant recipients, Task Force 2: Immonosuppression and Rejection. Nov 2010.

23. Colvin-Adams M, Agnihotri A. Cardiac allograft vasculopathy: current knowledge and future direction. Clin Transplant 2011; 25: 175.

24. Taylor DO, Stehlik J, Edwards LB, et al. Registry of the International Society for Heart and Lung Transplantation: Twenty-sixth Official Adult Heart Transplant Report-2009. J Heart Lung Transplant 2009; 28: 1007.

25. Petrakopoulou P, Anthopoulou L, Muscholl M, et al. Coronary endothelial vasomotor function and vascular remodeling in heart transplant recipients randomized for tacrolimus or cyclosporine immunosuppression. J Am Coll Cardiol 2006; 47: 1622.

26. Klauss V, Konig A, Spes C, et al. Cyclosporine versus tacrolimus (FK 506) for prevention of cardiac allograft vasculopathy. Am J Cardiol 2000; 85: 266.

27. The International society of heart and lung transplantation guidelines for the care of heart transplant recipients, Task Force 3: Long-term Care of Heart Transplant Recipients. Aug 2010.

28. Reichenspurner H. Overview of tacrolimus-based immunosuppression after heart or lung transplantation. J Heart Lung Transplant 2005; 24: 119.

29. Vassalli G, Gallino A, Weis M, et al. Alloimmunity and nonimmunologic risk factors in cardiac allograft vasculopathy. Eur Heart J 2003; 24: 1180.

30. Uretsky BF, Murali S, Reddy PS, et al. Development of coronary artery disease in cardiac transplant patients receiving immunosuppressive therapy with cyclosporine and prednisone. Circulation 1987; 76: 827.

31. Stoica SC, Cafferty F, Pauriah M, et al. The cumulative effect of acute rejection on development of cardiac allograft vasculopathy. J Heart Lung Transplant 2006; 25: 420.

32. Mehra MR, Crespo-Leiro MG, Dipchand A, et al. International Society for Heart and Lung Transplantation working formulation of a standardized nomenclature for cardiac allograft vasculopathy-2010. J Heart Lung Transplant 2010; 29: 717.

33. Wenke K, Meiser B, Thiery J, et al. Simvastatin initiated early after heart transplantation: 8-year prospective experience. Circulation 2003; 107: 93. 